\title{
Altered glycosylation of glycodelin in endometrial carcinoma
}

\author{
Laura C. Hautala ${ }^{1}$ Poh-Choo Pang ${ }^{2}$ Aristotelis Antonopoulos $\mathbb{D}^{2} \cdot$ Annukka Pasanen $^{3} \cdot$ Cheuk-Lun Lee $^{4}$. \\ Philip C. N. Chiu ${ }^{4} \cdot$ William S. B. Yeung ${ }^{4}$ Mikko Loukovaara ${ }^{5} \cdot$ Ralf Bützow $^{3,5} \cdot$ Stuart M. Haslam ${ }^{2} \cdot$ Anne Dell $^{2}$. \\ Hannu Koistinen $\mathbb{D}^{1}$
}

Received: 13 January 2020 / Revised: 18 February 2020 / Accepted: 18 February 2020 / Published online: 23 March 2020

(c) The Author(s) 2020. This article is published with open access

\begin{abstract}
Glycodelin is a major glycoprotein expressed in reproductive tissues, like secretory and decidualized endometrium. It has several reproduction related functions that are dependent on specific glycosylation, but it has also been found to drive differentiation of endometrial carcinoma cells toward a less malignant phenotype. Here we aimed to elucidate whether the glycosylation and function of glycodelin is altered in endometrial carcinoma as compared with a normal endometrium. We carried out glycan structure analysis of glycodelin expressed in HEC-1B human endometrial carcinoma cells (HEC-1B $\mathrm{Gd}$ ) by mass spectrometry glycomics strategies. Glycans of HEC-1B Gd were found to comprise a typical mixture of highmannose, hybrid, and complex-type N-glycans, often containing undecorated LacNAc (Gal $\beta 1-4 \mathrm{GlcNAc}$ ) antennae. However, several differences, as compared with previously reported glycan structures of normal human decidualized endometrium-derived glycodelin isoform, glycodelin-A (GdA), were also found. These included a lower level of sialylation and more abundant poly-LacNAc antennae, some of which are fucosylated. This allowed us to select lectins that showed different binding to these classes of glycodelin. Despite the differences in glycosylation between HEC-1B Gd and GdA, both showed similar inhibitory activity on trophoblast cell invasion and peripheral blood mononuclear cell proliferation. For the detection of cancer associated glycodelin, we established a novel in situ proximity-ligation based histochemical staining method using a specific glycodelin antibody and UEAI lectin. We found that the UEAI reactive glycodelin was abundant in endometrial carcinoma, but virtually absent in normal endometrial tissue even when glycodelin was strongly expressed. In conclusion, we established a histochemical staining method for the detection of endometrial carcinoma-associated glycodelin and showed that this specific glycodelin is exclusively expressed in cancer, not in normal endometrium. Similar methods can be used for studies of other glycoproteins.
\end{abstract}

Supplementary information The online version of this article (https:// doi.org/10.1038/s41374-020-0411-x) contains supplementary material, which is available to authorized users.

Hannu Koistinen

hannu.k.koistinen@helsinki.fi

1 Department of Clinical Chemistry, University of Helsinki and Helsinki University Hospital, Helsinki, Finland

2 Department of Life Sciences, Imperial College London, London, UK

3 Department of Pathology, University of Helsinki and Helsinki University Hospital, Helsinki, Finland

4 Department of Obstetrics and Gynaecology, The University of Hong Kong, Hong Kong SAR, China

5 Department of Obstetrics and Gynecology, University of Helsinki and Helsinki University Hospital, Helsinki, Finland

\section{Introduction}

Glycodelin (also known as Progestagen Associated Endometrial Protein, PAEP, and Placental Protein 14, PP14) is a secreted human lipocalin-family protein mainly expressed in well-differentiated epithelial cells in human reproductive tissues, like endometrium and seminal vesicles $[1,2]$. In premenopausal endometrium the expression of glycodelin is temporally regulated by progesterone, expression increasing shortly after ovulation until the end of the menstrual cycle. Depending on the cell of origin glycodelin is differently glycosylated, resulting in glycodelin isoforms with different biological actions [1, 3-8]. We have previously characterized four types of glycodelin, which are derived from different reproductive tissues: decidualized endometriumderived glycodelin-A (GdA, isolated from amniotic fluid), glycodelin-F (isolated from follicular fluid), glycodelin-C (isolated from cumulus cells), and seminal vesicle-derived 
glycodelin-S (GdS, isolated from seminal plasma) $[5,7,9,10]$. Each of these expresses a characteristic glycan profile. This translates to differences in functional assays. Thus, while glycodelin-A inhibits sperm-egg binding, i.e., fertilization, glycodelin-S prevents premature sperm capacitation, but does not prevent sperm-egg binding [10, 11]. In contrast, glycodelin-F prevents premature acrosome reaction of sperm, whilst glycodelin-C, which is believed to be derived from glycodelin-F via glycan processing by cumulus cells, stimulates sperm-egg binding [4, 12, 13]. Thus, it appears that different glycodelin isoforms participate in a cascade of activities regulating the sperm functions needed for successful fertilization. Furthermore, immunosuppressive activities of glycodelin have been ascribed to glycosylation [5, 14]. These include the inhibition of peripheral blood mononuclear cell (PBMC) proliferation $[5,15]$. We have previously also reported that glycodelin-A inhibits trophoblast invasion, while deglycosylated glycodelin or differentially glycosylated glycodelin-F does not have this activity [16]. Tight regulation of trophoblast invasion is essential for successful pregnancy [17]. Based on these studies, glycodelin is considered as a remarkable example of how glycosylation may dictate the functions of glycoproteins [18].

We have suggested that glycodelin also acts as a tumor suppressor protein, at least in endometrial carcinoma, based on our findings showing that glycodelin transfection in HEC-1B human endometrial carcinoma cells cause morphological reversion from malignant phenotype to a more normal state [19-21]. This is mediated by a repressed PKC$\delta$ activation [21]. Furthermore, in a preclinical mouse model, glycodelin-induced differentiation brought about reduced tumor growth [20]. Further proof for the inhibitory role of glycodelin in malignant transformation comes from the studies in which reversion of Ishikawa endometrial adenocarcinoma cells to resemble normal endometrial epithelial cells was induced by histone deacetylase inhibitors [22]. This differentiation was accompanied by glycodelin synthesis. Importantly, knockdown of glycodelin expression by RNA-interference blocked the malignant-to-normal reversion, demonstrating that glycodelin is crucial in this process. Glycodelin transfection into these cells caused reduced cell proliferation [23]. Malignant growth is associated with loss of cell differentiation, and many tumor suppressor genes that protect cells against malignant transformation are known to regulate cell differentiation [24]. Therefore it is not surprising that the expression of glycodelin is reduced in hormone-related cancers, including ovarian and breast cancers, and further reduced in less differentiated tumors [25-27].

Complex glycosylation, which greatly increases the structural diversity of glycoproteins, is essential for multicellular organisms [28]. Despite the utmost importance of protein glycosylation, the human glycome is not well characterized, mainly due to complexity of carbohydrate structures. Changes in protein glycosylation have been observed in several human cancers, where they affect tumorigenic properties, like cellular growth behavior, invasiveness and acquisition of metastatic potential [29-32]. Although many glycodelin isoforms have been characterized from reproductive tissues, nothing is known about the glycosylation of glycodelin produced by endometrial carcinoma tissue. Here, with the aid of glycomics analysis, we established a novel glycodelin-glycoformspecific histochemical staining method and showed that glycodelin is differentially glycosylated in endometrial carcinoma tissue as compared with normal endometrium.

\section{Material and methods}

\section{Production and purification of glycodelin}

HEC-1B cells (ATCC HTB-113), which do not normally produce glycodelin, were stably transfected with glycodelin cDNA [20, 21]. This cell line is a subline [33] of HEC-1 cells derived from a moderately differentiated papillary adenocarcinoma tumor from a patient with stage IA endometrial cancer [34]. The cells were cultured in RPMI-1640 (Lonza), supplemented with $10 \%$ fetal calf serum (FCS), $100 \mathrm{IU} / \mathrm{ml}$ penicillin, $100 \mu \mathrm{g} / \mathrm{ml}$ streptomycin and $2 \mathrm{mM}$ L-glutamine, at $37{ }^{\circ} \mathrm{C}$ in humidified incubator with $5 \% \mathrm{CO}_{2}$. The cell clones were authenticated as HEC-1B cells using microsatellite markers (Promega GenePrint 10 System) by the Institute for Molecular Medicine Finland (FIMM) Technology Centre, University of Helsinki.

Glycodelins from HEC-1B cell culture medium (HEC1B Gd) and pooled amniotic fluids (glycodelin-A, GdA) were purified using a monoclonal anti-glycodelin antibody (F43-7F9) column as described previously [35]. Before purification Triton X-100 (0.1\% final concentration) was added to the cell culture medium or amniotic fluid. After affinity purification, buffer was changed to PBS and any detached antibodies were removed using protein $\mathrm{G}$ column. Glycodelin preparations were concentrated using a centrifugal filter device (Amicon Ultra-4, 10,000 NMWL) and the concentrations were measured by an immunoassay [36].

\section{Glycomics analysis by MALDI-TOF/TOF and data interpretation}

Glycomics analysis was performed using strategies previously optimized for GdA characterization [5, 7, 37]. Purified HEC-1B Gd (50 $\mu \mathrm{g})$ was digested using sequencing grade trypsin (Promega). The N-glycans were then released 
Table 1 Lectin-immunoassay results.

\begin{tabular}{|c|c|c|c|c|}
\hline \multirow[t]{2}{*}{ Lectin $^{a}$} & \multirow[t]{2}{*}{ Abbreviation } & \multicolumn{2}{|c|}{ Reaction with ${ }^{b}$} & \multirow[t]{2}{*}{ Specificity $^{\mathrm{c}}$} \\
\hline & & GdA & HEC-1B Gd & \\
\hline Sambucus nigra & SNA & +++ & - & Neu5Ac $\alpha 6 \mathrm{Gal} / \mathrm{GalNAc}$ \\
\hline Erythrina cristagalli & ECL & - & +++ & Gal $\beta 4 G l c N A c$ \\
\hline Ulex europaeus I & UEAI & - & +++ & $\alpha$ Fuc \\
\hline Concanavalin A & Con-A & + & +++ & $\alpha$ Man, $\alpha$ Glc \\
\hline Pisum sativum & PSA & + & + & $\alpha \operatorname{Man}, \alpha \mathrm{Glc}$ \\
\hline Lens culinaris & LCA & + & +++ & $\alpha$ Man, $\alpha$ Glc \\
\hline Ricinus communis I & RCA I & + & + & Gal \\
\hline Griffonia simplicifolia II & GSL II & - & - & $\alpha \mathrm{Gal}$ \\
\hline Griffonia simplicifolia I & GSL I & - & - & $\alpha \mathrm{Gal}, \alpha \mathrm{GalNAc}$ \\
\hline Dolichos biflorus & DBA & - & - & $\alpha$ GalNAc \\
\hline Soybean & SBA & + & +++ & $\alpha>\beta$ GalNAc \\
\hline Sophora japonica & SJA & - & - & $\beta$ GalNAc \\
\hline Vicia villosa & VVA & - & - & GalNAc \\
\hline Wistera floribunda & WFA & + & ++ & GalNAc \\
\hline Wheat Germ & WGA & + & ++ & GlcNAc \\
\hline Succinylated Wheat Germ & WGA $_{\text {succ }}$ & + & +++ & GlcNAc \\
\hline Datura stramonium & DSL & - & - & $(\mathrm{GlcNAc})_{2-4}$ \\
\hline Lycopersicon esculentum $^{\mathrm{d}}$ & LEL & - & - & $(\mathrm{GlcNAc})_{2-4}$ \\
\hline Solanum tuberosum & STL & + & + & $(\mathrm{GlcNAc})_{2-4}$ \\
\hline Peanut & PNA & - & - & Gal $\beta 3$ GalNAc \\
\hline Jacalin & Jacalin & - & - & Gal $\beta 3$ GalNAc \\
\hline Phaseolus vulgaris Erythroagglutinin & PHA-E & - & - & $\begin{array}{l}\text { Gal } \beta 4 \text { GlcNAc } \beta 2 \text { Man } \alpha 6 \\
\text { (GlcNAc } \beta 4) \\
(\text { GlcNAc } \beta 4 \text { Man } \alpha 3) \\
\text { Man } \beta 4\end{array}$ \\
\hline Phaseolus vulgaris Leucoagglutinin & PHA-L & + & + & $\begin{array}{l}\text { Gal } \beta 4 \text { GlcNAc } \beta 6(\mathrm{GlcNAc} \\
\beta 2 \text { Man } \alpha 3) \text { Man } \alpha 3\end{array}$ \\
\hline
\end{tabular}

${ }^{a}$ Lectin from Wisteria floribunda (WFA, L-1516) was obtained from Sigma. Other lectins were from Vector Laboratories as Biotinylated Lectin Kits I, II, and III.

${ }^{\mathrm{b}}-$, no reactivity (signal $<1.5$-fold of that of blank, i.e., signal obtained with biotinylated lectin and labeled antibody without GdA or HEC-1B Gd); +, positive signal ( $>1.5$-fold blank);,$++>2$ - and $<4$-fold difference between the reactivity with GdA and HEC-1B Gd (after deduction of blank value);,$+++>4$-fold difference between the reactivity with GdA and HEC-1B Gd. The relative reactivity between different lectins cannot be deduced from the table.

'Specificity is based on the manufacturer's (Vector Laboratories) information.

${ }^{\mathrm{d}}$ High background signal (perhaps due to direct binding of the detection antibody to the lectin). by N-glycosidase F (Roche Applied Science) and separated on Sep-Pak $\mathrm{C} 18$ cartridges. The native $\mathrm{N}$-glycans were permethylated using the sodium hydroxide procedure, subjected to chloroform extraction, and a further clean-up step using Sep-Pak C18 cartridges. The purified permethylated $\mathrm{N}$-glycans were then dissolved in methanol and an aliquot of the sample was mixed with a 3,4-diaminobenzophenone matrix solution $(10 \mathrm{mg} / \mathrm{ml}$ in $75 \%$ acetonitrile/water) (Acros Organics) at a 1:1 ratio $(\mathrm{v} / \mathrm{v})$. The glycan-matrix mixture was spotted on a MALDI plate and allowed to dry and crystallize. MALDI-TOF and -TOF/TOF (MS and MS/MS) data were obtained using a 4800 MALDI-TOF/TOF mass spectrometer (AB Sciex UK
Limited) in positive ion mode. Argon was used as the collision gas with collision energy of $1 \mathrm{kV}$.

The MALDI MS and MS/MS data obtained were analyzed using Data Explorer 4.9 (AB Sciex UK Limited). Manual assignment of glycan sequences was based on the known biosynthetic pathways, with the aid of the glycobioinformatics tool, GlycoWorkBench [38].

\section{Lectin immunoassays}

Lectin immunoassays were carried out essentially as previously described [36], using biotinylated lectins obtained from Vector Laboratories or from Sigma (Table 1). Briefly, 
biotinylated lectins were captured on streptavidin coated wells (96-well plate; PerkinElmer). In the assay, $25 \mu \mathrm{l}$ sample (dilutions of HEC-1B Gd and GdA) and $200 \mu \mathrm{l}$ assay buffer, supplemented with $1 \mathrm{mM} \mathrm{CaCl}, \mathrm{MnCl}_{2}$, and $\mathrm{MgCl}_{2}$, were incubated overnight at $4{ }^{\circ} \mathrm{C}$ in wells. After washing the wells Eu-labeled antibody against glycodelin (F25-9D8) was added for $2 \mathrm{~h}$ at room temperature. After washing enhancement solution was added and the fluorescence was measured.

\section{Invasion assays}

Human placental tissue was obtained with a written consent from women who underwent surgical termination of pregnancy in the first trimester. Primary trophoblast expressing CK7 and HLAG were obtained as described [39, 40]. Briefly, the placental villi were digested with trypsin and DNAase, filtered through 100 and $40 \mu \mathrm{m}$ filters, and centrifuged by using density gradient media (Ficoll-Paque). The isolated cells were then incubated in a plastic culture dish for $20 \mathrm{~min}$ to remove adherent leukocytes. Nonadherent cells were seeded onto fibronectin-coated plates and incubated overnight.

The adherent primary trophoblasts and JEG-3 human choriocarcinoma cells (ATCC HTB-36) were cultured in Dulbecco's Modified Eagle Medium and Eagle's Minimum Essential Medium (EMEM) (Lonza), respectively, supplemented with $10 \%$ FCS, $100 \mathrm{IU} / \mathrm{ml}$ penicillin, $100 \mu \mathrm{g} / \mathrm{ml}$ streptomycin, and $2 \mathrm{mM}$ L-glutamine, at $37^{\circ} \mathrm{C}$ in a humidified, $\mathrm{CO}_{2}$-controlled $(5 \%)$ incubator. Primary trophoblasts $\left(3 \times 10^{5}\right)$ and JEG-3 cells $\left(1.5 \times 10^{5}\right)$ in serum free medium containing 0 or $10 \mu \mathrm{g} / \mathrm{ml}$ either GdA or HEC-1B Gd were added into Polycarbonate Membrane Transwell ${ }^{\circledR}$ inserts with $8.0 \mu \mathrm{m}$ pore size (Corning, Inc.) covered with $100 \mu \mathrm{l}$ phenolred free Matrigel $^{\mathrm{TM}}$ Basement Membrane Matrix (BD Biosciences) diluted 1:2 with EMEM. The inserts were placed into 24-well plate containing $400 \mu \mathrm{l}$ of culture medium with $10 \%$ FCS and incubated for $22 \mathrm{~h}$. The Matrigel and the non-invasive cells were removed using cotton swabs and the membranes were stained with $0.09 \%$ crystal violet for $10 \mathrm{~min}$. The cells were counted under a microscope and the mean of three fields per membrane was calculated.

\section{$\mathrm{XTT}$ cell viability assays}

Human female peripheral blood was obtained from the Hong Kong Red Cross. PBMCs were isolated by density gradient centrifugation (Ficoll-Paque). Cell viability was determined by the XTT assay (Roche Diagnostics) according to the manufacturer's protocol. In brief, PBMCs $\left(3 \times 10^{4}\right)$ were incubated with $0.01,0.1,1$, and $10 \mu \mathrm{g} / \mathrm{ml}$ glycodelins for $36 \mathrm{~h}$, and XTT-labeling mixture was added $12 \mathrm{~h}$ before absorbance measurement at 450 and $595 \mathrm{~nm}$.
The cell viability was expressed as relative viability (\%) using the following equation:

$$
\begin{aligned}
\text { Relative viability }(\%)= & (\text { Abs GdA }- \text { Abs blank }) / \\
& (\text { Abs control }- \text { Abs blank }) \times 100 \% .
\end{aligned}
$$

\section{Histochemical stainings}

Formalin-fixed, paraffin-embedded tissue samples were collected at Pathology Department, the Helsinki University Hospital, Helsinki, Finland. Tissue microarrays (TMA) including 75 endometrial carcinomas of the endometrioid subtype and 52 samples of normal control endometrium were prepared as described previously [41]. The clinicopathological data of the patients selected for this study are shown in Table 2. Three secretory phase endometrium samples served as positive controls. Approvals of the Institutional Review Board of the Helsinki University Hospital and the National Supervisory Authority for Welfare and Health were obtained.

Five $\mu \mathrm{m}$ thick sections were cut from the tissue and TMA blocks and placed onto glass slides. For histochemical stainings (below), the sections were deparaffinized and rehydrated. Stainings were scored by an experienced pathologist (A.P.).

\section{Glycodelin staining}

To enhance the antigen retrieval the sections were heated in citrate buffer ( $\mathrm{pH}$ 6.0) in a microwave oven for $10 \mathrm{~min}$. This was followed by the addition of peroxidase-blocking solution (Dako REAL, \#S2023, DAKO) for $30 \mathrm{~min}$ and horse serum (diluted 1:20 in PBS, Vector Laboratories) for $20 \mathrm{~min}$, both at room temperature. Rabbit (\#1) antiglycodelin $\operatorname{IgG}$ [25] was used as the primary antibody $\left(3 \mu \mathrm{g} / \mathrm{ml}\right.$, incubated for overnight at $\left.+4{ }^{\circ} \mathrm{C}\right)$ and the $\operatorname{IgG}$ fraction from preimmune serum of the same rabbit $(3 \mu \mathrm{g} / \mathrm{ml})$ was used as a negative control. Labeled polymer-HRP antirabbit (DAKO, K4009) was used as a secondary detection reagent (30 $\mathrm{min}$ at room temperature). Histofine Simple Stain AEC Solution (Nicherei Biosciences) was used as a chromogen for antigen detection. Counterstaining was performed with modified Mayer's staining solution (\#01820, Histolab Products Ab). For the progesterone receptor (PR) staining, $3 \mu \mathrm{m}$ sections were stained with Ventana Benchmark Ultra (Roche/Ventana). Heat-induced epitope retrieval was performed using standard pretreatment buffer $\mathrm{CC} 1$ (Roche/Ventana), $64 \mathrm{~min} / 98^{\circ} \mathrm{C}$. The slides were incubated with PR antibody (dilution 1:50, clone 16, NCL-L-PGR-312, Novocastra) $32 \mathrm{~min} / 37^{\circ} \mathrm{C}$. For detection we used multimer-based detection kit Optiview (760-700, Roche/Ventana). The slides were counterstained with Mayer's hematoxylin, and were then dehydrated and 
Table 2 Clinicopathological data in relation to glycodelin (Gd), UEAI lectin (UEAI) and UEAI-glycodelin (UEAI-Gd) staining.

\begin{tabular}{|c|c|c|c|c|}
\hline & $n(\%)^{\mathrm{b}}$ & $\begin{array}{l}\text { Gd pos. } \\
n(\%)^{\mathrm{c}}\end{array}$ & $\begin{array}{l}\text { UEAI pos. } \\
n(\%)^{\mathrm{c}, \mathrm{d}}\end{array}$ & $\begin{array}{l}\text { UEAI-Gd } \\
\text { pos } n(\%)^{\mathrm{c}}\end{array}$ \\
\hline $\begin{array}{l}\text { Endometrial } \\
\text { carcinoma }^{\mathrm{a}}\end{array}$ & $75(100)$ & $22(29)$ & $57(77)$ & $36(48)$ \\
\hline \multicolumn{5}{|l|}{ Grade } \\
\hline $1-2$ & $65(87)$ & $21(32)$ & $51(78)$ & $33(51)$ \\
\hline 3 & $10(13)$ & $1(10)$ & $6(67)$ & $3(30)$ \\
\hline$p$ value $\mathrm{e}^{\mathrm{e}}$ & & $p=0.26$ & $p=0.42$ & $p=0.31$ \\
\hline \multicolumn{5}{|l|}{ Stage } \\
\hline I & $62(83)$ & 18 (29) & $48(79)$ & $29(47)$ \\
\hline II-IV & $13(17)$ & $4(31)$ & $9(69)$ & $7(54)$ \\
\hline$p$ value $^{\mathrm{e}}$ & & $p=1.0$ & $p=0.48$ & $p=0.76$ \\
\hline \multicolumn{5}{|l|}{ Tumor size ${ }^{f}$} \\
\hline Diameter $<2 \mathrm{~cm}$ & $22(30)$ & $7(32)$ & $16(73)$ & $11(50)$ \\
\hline Diameter $\geq 2 \mathrm{~cm}$ & $51(70)$ & $14(27)$ & $39(78)$ & $24(47)$ \\
\hline$p$ value $\mathrm{e}^{\mathrm{e}}$ & & $p=0.70$ & $p=0.49$ & $p=0.69$ \\
\hline \multicolumn{5}{|l|}{$\mathrm{LVI}^{\mathrm{f}}$} \\
\hline Yes & $17(24)$ & $6(35)$ & $12(71)$ & $7(41)$ \\
\hline No & $55(76)$ & 15 (27) & $43(80)$ & $27(49)$ \\
\hline$p$ value $^{\mathrm{e}}$ & & $p=0.80$ & $p=0.86$ & $p=1.0$ \\
\hline $\begin{array}{l}\text { Normal } \\
\text { endometrium }^{\mathrm{a}}\end{array}$ & $52(100)$ & $6(12)$ & $17(34)$ & $1(2)$ \\
\hline $\begin{array}{l}p \text { value (cancer } \\
\text { vs normal) }^{\mathrm{e}}\end{array}$ & & $p=0.018$ & $p<0.0001$ & $p<0.0001$ \\
\hline
\end{tabular}

${ }^{\mathrm{a}}$ The mean $\pm \mathrm{SD}$ age of patients was $65.7 \pm 11.3$ years (range $37-92$ years) and for controls $57.9 \pm 6.9$ years (range $38-77$ ).

${ }^{\mathrm{b}}$ Number of samples in each category and in parenthesis percent.

${ }^{\mathrm{c}}$ Number and in parenthesis percent of positively stained samples in each category.

${ }^{\mathrm{d}}$ In UEAI staining one endometrioid carcinoma (grade 3, stage I, diameter $\geq 2 \mathrm{~cm}$ and no LVI) and two control samples were missing.

e $p$ values (Fisher's exact test, two-sided) between frequency of positively stained samples in different subgroups or between carcinoma and normal endometrium.

${ }^{\mathrm{f}}$ Tumor size or lymphovascular invasion (LVI) were not available from 2 or 3 patients, respectively.

mounted for viewing with microscope. Glycodelin staining was scored either strong positive, weak/focal positive, or negative. PR staining was scored either positive or negative.

\section{Glycodelin-UEAI-lectin in situ proximity ligation assay $\left(\right.$ PLA $\left.^{\circledR}\right)$}

Unless otherwise indicated, in situ $\mathrm{PLA}^{\circledR}$ was performed using the Duolink ${ }^{\circledR}$ reagents essentially according to manufacturer's instructions (Sigma-Aldrich). Briefly, endogenous peroxidase activity was blocked by $30 \mathrm{~min}$ treatment with $0.3 \% \mathrm{H}_{2} \mathrm{O}_{2}$ (DUO82054) at room temperature. Tissue slices were blocked using blocking solution (DUO82007) for $30 \mathrm{~min}$ at $37^{\circ} \mathrm{C}$. Monoclonal mouse antibody against glycodelin [3 $\mu \mathrm{g} / \mathrm{ml}$, F25-9D8 [36]] and biotinylated Ulex europaeus agglutinin I (UEAI) $(5 \mu \mathrm{g} / \mathrm{ml}$, Vector Laboratories) were diluted in antibody diluent (DUO82008) and incubated with sections overnight at $37^{\circ} \mathrm{C}$. Duolink in situ PLA Probe Anti-Mouse PLUS (DUO92001) and streptavidin $(2 \mu \mathrm{g} / \mathrm{ml})$ conjugated with Duolink In Situ probemaker MINUS (DUO92010) were used as detection probes. The reaction with probes, ligation, amplification $\left(2 \mathrm{~h}\right.$ at $\left.37^{\circ} \mathrm{C}\right)$, and detection were performed according to the manufacturer's instructions. The luminal and cytoplasmic staining were scored either positive or negative.

\section{Lectin staining}

Endogenous peroxidase activity was blocked using peroxidase-blocking solution (Dako REAL, \#S2023) for 30 min, followed by incubation with horse serum (diluted 1:20 in PBS, Vector Laboratories) for $20 \mathrm{~min}$, both at room temperature. Biotinylated UEAI lectin $(25 \mu \mathrm{l} / \mathrm{ml}$ in PBS containing $1 \%$ BSA, $1 \mathrm{mM} \mathrm{CaCl}, 1 \mathrm{mM} \mathrm{MgCl}_{2}, 1 \mathrm{mM}$ $\mathrm{MnCl}_{2}$ ) was incubated overnight at $+4{ }^{\circ} \mathrm{C}$. $\mathrm{ABC}$ kit (PK6100 , Vector Laboratories) was used as a secondary detection reagent according to manufacturer's instructions. Staining with AEC and Mayer's staining solution were performed as in glycodelin staining. Staining in carcinoma cells and epithelial cells of normal endometrium was scored either positive or negative ( $<10 \%$ of cells stained). Blood vessels were not included in the evaluation.

\section{Data analyses}

Fisher's exact test (two-sided, IBM SPSS Statistics 24) was used to evaluate associations between different patient groups and staining. $T$-test (two-sample unequal variance, two-sided, Microsoft Excel) was used to evaluate differences in biological activity of different glycodelin preparations.

\section{Results}

\section{Glycomics analysis of glycodelin expressed by HEC-1B cells (HEC-1B Gd)}

Glycomics analysis was performed using strategies previously optimized for GdA characterization [5, 7]. The permethylated N-glycans were subjected to MALDI-MS profiling and MALDI-MS/MS sequencing. The complete MALDI-MS spectrum of the N-glycans of HEC-1B Gd and annotation of the most abundant informative molecular ions is shown in Fig. 1a. Comprehensive annotations are shown on the magnified spectrum in Supplementary Fig. The glycan structures were manually assigned by combining 

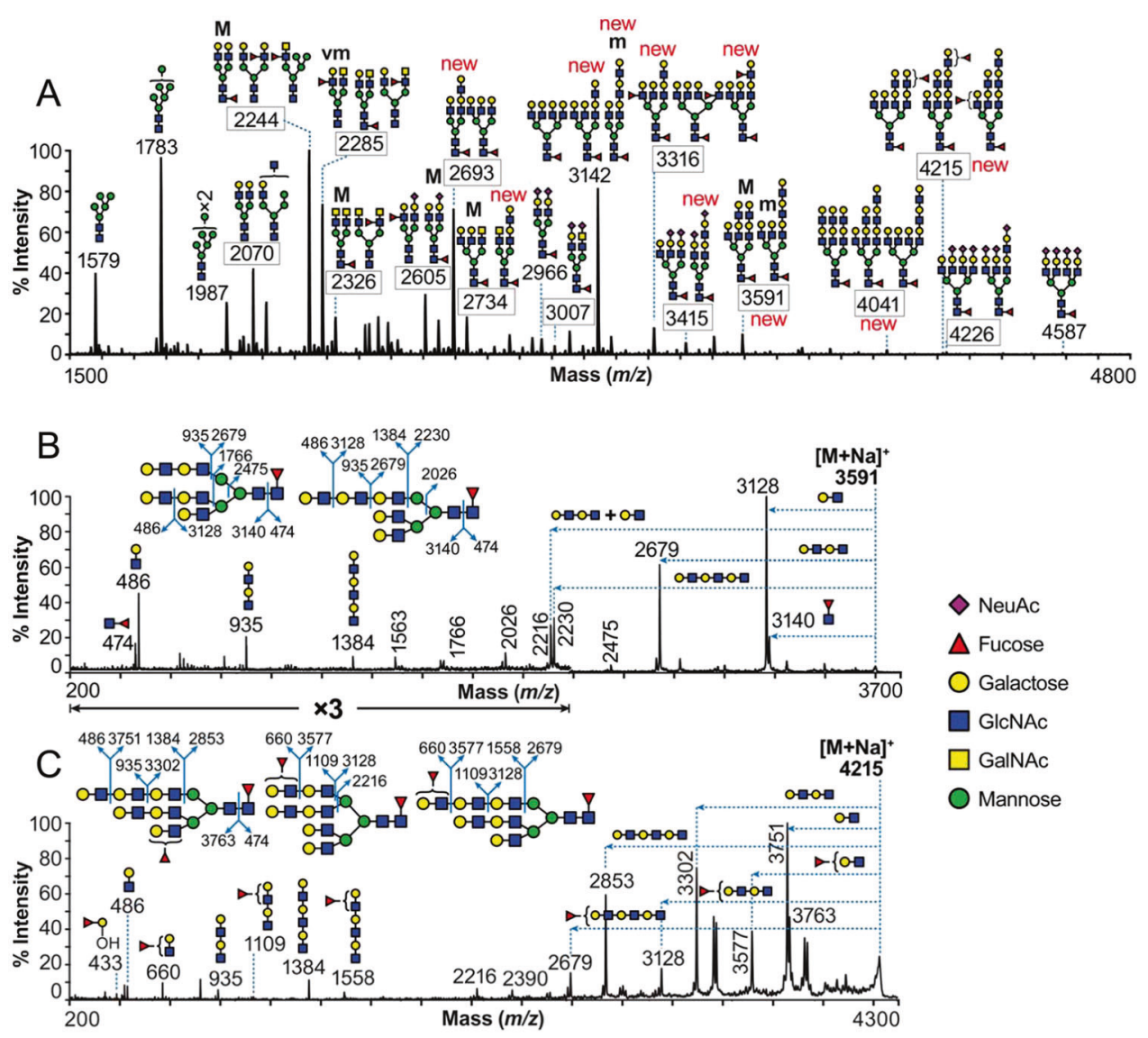

Fig. 1 Glycomics analysis of glycodelin expressed by HEC-1B cells (HEC-1B Gd). a MALDI-TOF mass spectra of permethylated Nglycans from HEC-1B Gd. "new" above particular structure, or next to a $\mathrm{m} / \mathrm{z}$ value, indicates new structure or a set of structures when compared with the $\mathrm{N}$-glycome of GdA analyzed before [5]. All signals observed are singly charged sodiated ions $[\mathrm{M}+\mathrm{Na}]^{+}$, and their structural assignment is based on monosaccharide composition, MS/ MS fragmentation analyses (boxed $\mathrm{m} / \mathrm{z}$ values) and knowledge of the glycan biosynthetic pathways. Fully annotated spectra that have been expanded on the $m / z$ axis to enable all components to be visualized, are presented in Supplementary Fig. Sugars shown on top of a bracket have not had their antenna location unequivocally defined. For

information on the $\mathrm{N}$-glycan compositions and knowledge of human N-glycan biosynthetic pathways. These assignments were then further confirmed and/or corrected based on MS/MS data, which were also manually interpreted. For practical reasons not all molecular ions in the spectra could be sequenced by MALDI-TOF/TOF MS/MS. Nonetheless it was possible to sequence representative members of each family of glycans as well as molecular ions not previously reported in GdA. Molecular ions selected for MS/MS are indicated with boxed $m / z$ values in Supplementary Fig.

Overall, the HEC-1B Gd glycan population comprised of a mixture of high-mannose, hybrid, truncated, and abundant complex $\mathrm{N}$-glycans that is typical of other female glycodelins (Fig. 1a). The most abundant (in terms of simplicity, one branching pattern for tri-antennary structures is shown. Therefore, the position of an antenna in a cartoon does not imply designation of a specific arm. "M", " $\mathrm{m}$ " and " $\mathrm{vm}$ " designations indicate major, minor and very minor abundances, respectively. b, $\mathbf{c}$ Evidence for LacNAc extension of N-glycans. MALDI-TOF/TOF MS/MS of the molecular ion at (b) $\mathrm{m} / \mathrm{z}, 3591$ and (c) 4215 as detected on the MALDI-TOF MS spectrum (see a). The fragment ions are consistent with the sequences shown in corresponding insets (major abundance structures). The horizontal arrows on the spectra indicate losses of glycan moieties from the molecular ion. c The fucose residues outside the bracket have not had their location unequivocally defined.

relative abundance) complex $\mathrm{N}$-glycans corresponded mainly to core-fucosylated structures with undecorated Gal $\beta 1-4 G l c N A c$ (LacNAc) units on their antennae $(\mathrm{m} / \mathrm{z}, 2244,2693,3142,3591$, and 4041). N-glycans with undecorated GalNAc $\beta 1-4$ GlcNAc (LacdiNAc) units, also mainly core-fucosylated, were also detected $(\mathrm{m} / \mathrm{z}, 2285$, 2326 and 2734), but in minor abundance. Both the LacNAc and LacdiNAc containing N-glycans were occasionally decorated by NeuAc $(\mathrm{m} / \mathrm{z} 2605,2966,3007,3415,4226$, and 4587$)$ or fucose $(\mathrm{m} / \mathrm{z} 2244,2285,2326,2605,3316$, and 4215) residues. Comprehensive analysis of the molecular ions, the composition of which corresponded to multiples of LacNAc units (e.g., $\mathrm{m} / \mathrm{z}$ 3142, 3316, 3415, 3591,4041 , and 4215), indicated that they were actually a 
Fig. 2 Biological activity of HEC-1B Gd and GdA.

a Trophoblast invasion assays were performed using primary trophoblasts $(n=3)$ and JEG-3 choriocarcinoma cells $(n=6)$. b Peripheral blood mononuclear cell (PBMC) viability was evaluated by XTT assay $(n=4)$ $* p<0.05$ or $* * p<0.01$ vs. control ( $T$-test). Data represent mean $\pm \mathrm{SE}$
A

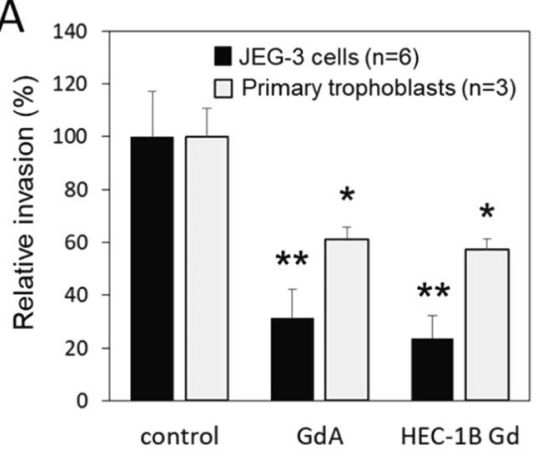

B

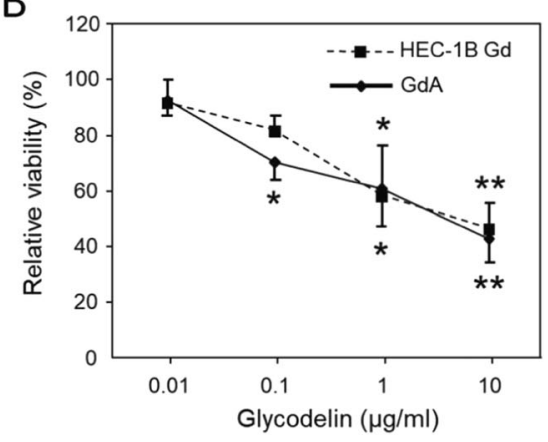

mixture of isomers of which a minority contained linear poly-LacNAc antennae. Two examples are the structures detected for the molecular ions at $\mathrm{m} / \mathrm{z}, 3591$ and 4215 . MALDI-TOF/TOF MS/MS of the former molecular ion exhibited fragment ions that corresponded to losses of two and three LacNAc repeating units $(\mathrm{m} / z, 2679$ and 2230, respectively, Fig. 1b) indicating the presence of linear polyLacNAc units. Similarly, the molecular ion at the $\mathrm{m} / \mathrm{z} 4215$ exhibited fragment ions that corresponded to losses of two and three LacNAc repeating units, each further modified with a fucose residue $(\mathrm{m} / \mathrm{z} 3128$ and 2679 , respectively, Fig. 1c). The detection of the fragment ions at $\mathrm{m} / z 433$ and 660 , indicated that in certain cases the fucose residue was at the non-reducing terminus of the above poly-LacNAc units. Particularly, the presence of fragment ion at $\mathrm{m} / \mathrm{z} 433$, together with the absence of a fragment ion at $\mathrm{m} / \mathrm{z} 834$ (indicating the absence of $\mathrm{Le}^{\mathrm{b} / \mathrm{y}}$ epitopes for the specific molecular ion), suggested the presence of $\mathrm{H}$ type antigen (Fuc $\alpha 1-2$ Gal $\beta 1-3 / 4$ GlcNAc $\beta$ ).

As compared with previously reported $\mathrm{N}$-glycans of GdA $[5,7]$, the above N-glycan population of HEC-1B Gd exhibited differences with the most noticeable being the following: (i) the reduced abundance of Sda (NeuAco2-3 [GalNAc $\beta 1-4] \mathrm{Gal}$ ) epitope (detected only at $\mathrm{m} / \mathrm{z}$ 2646, Supplementary Fig.); (ii) the reduced abundance of sialylated N-glycans; and (iii) the increased abundance of linear poly-LacNAc units that were further modified with NeuAc or fucose residues.

\section{Reactivity with lectins}

The glycan-analyses data allowed us to select lectins that potentially show differential binding to GdA and HEC-1B Gd. Also, several other lectins were tested (Table 1). The lecting binding studies confirmed the differences between the glycans of HEC-1B Gd and GdA. We found that UEAI and Erythrina cristagalli agglutinin (ECL) exclusively recognized HEC-1B Gd, not GdA, while Sambucus nigra agglutinin (SNA) recognized only GdA. Several other lectins bound preferentially to HEC-1B Gd but bound weakly also to GdA.

\section{Bioactivity of HEC-1B Gd}

The bioactivity of HEC-1B Gd was studied by assays in which we previously have found glycosylation to be important for the activity of glycodelin $[5,7,16]$. Trophoblast invasion assays were conducted both using primary trophoblast cells and JEG-3 choriocarcinoma cells. Irrespective of the differential glycosylation of HEC-1B Gd and $\mathrm{GdA}$, these glycodelin isoforms had similar inhibitory activity on trophoblast cell invasion (Fig. 2a). The same was observed in inhibition of PBMC viability (Fig. 2b).

\section{Histochemical staining of glycodelin and its UEAI reactive glycoform in endometrial carcinoma tissue}

To test whether the differences found in endometrial carcinoma cell line (HEC-1B Gd) and endometrium (GdA) derived glycodelins are also found in endometrium tissue of cancer patients and controls, we established a specific staining method for UEAI lectin-reactive glycodelin (UEAI-glycodelin).

First, we stained glycodelin with an antibody that does not discriminate between the glycoforms. In TMA, the amount of samples with strong glycodelin staining was not different between the endometrial carcinoma $(5.3 \%)$ and control groups $(5.8 \%)$ ( $p=1.0$, Fisher's exact test), while the overall glycodelin staining (i.e., both strong and weak focal staining) was more frequent in carcinoma group ( $29.3 \%$ vs. $11.5 \%, p=0.018$ ) (Fig. 3, Table 2). In histologically normal endometrium, glycodelin staining was found in glandular epithelium, while in tumoral tissue glycodelin was localized in the carcinoma cells (Fig. 3a-d, g). However, even the strong staining in the carcinoma samples tended to be weaker and more focal than that in non-malignant endometrial tissue, including three histological sections of the secretory phase endometrium, which showed very strong glycodelin staining (Fig. 3a). Interestingly, three endometrial samples in which areas of both cancerous and histologically normal endometrium were present showed strong glycodelin staining only in nonmalignant area, while cancerous regions in the same 

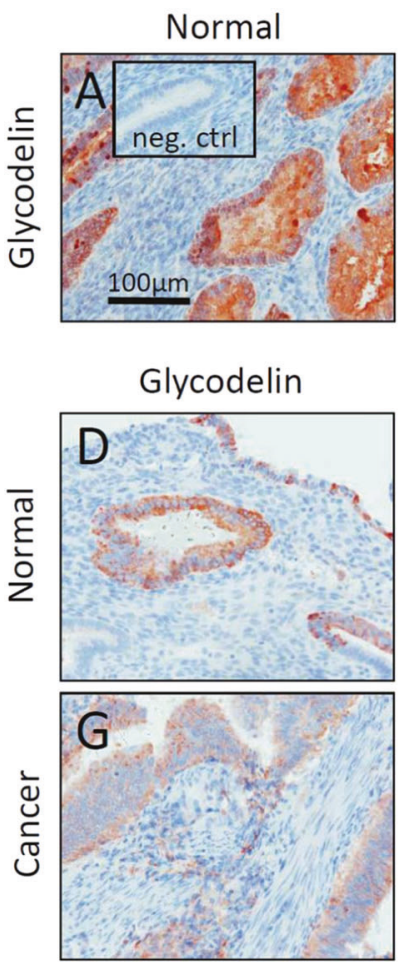

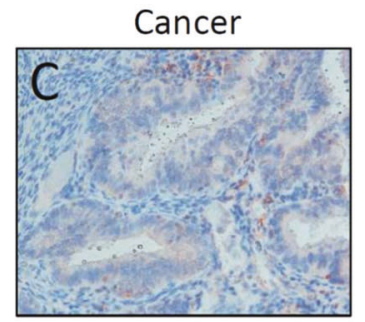

UEAI-glycodelin
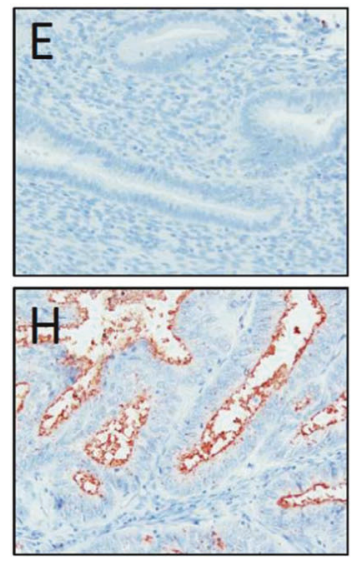

UEAI
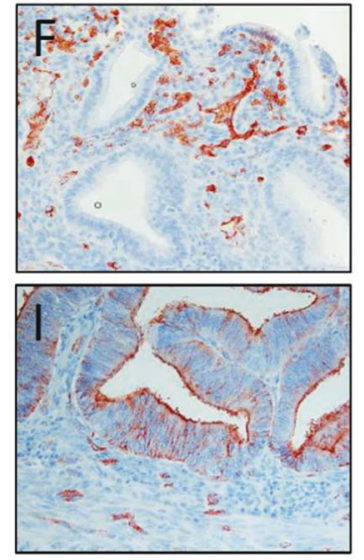

Fig. 3 Histochemical staining of glycodelin, UEAI lectin-reactive glycodelin (UEAI-glycodelin) and UEAI-binding glycans in normal endometrium and endometrial carcinoma tissues. Example of glycodelin staining in a whole tissue section (b), which has both cancerous region (c) and normal secretory endometrium (a). Insert in $\mathbf{a}$ is a negative control in which the glycodelin antibody has been replaced with preimmune IgG. Representative examples of strong

sections were glycodelin negative or only weakly stained (Fig. 3a-c).

In in situ proximity ligation based staining of UEAI lectin-reactive glycodelin (UEAI-glycodelin) $30.7 \%$ of the endometrioid carcinoma tissues showed luminal staining, $1.3 \%$ cytoplasmic staining, and $16.0 \%$ both cytoplasmic and luminal staining (Fig. 3e, h, Table 2). The noncancerous tissues showed positive staining only in one case $(p<0.001$ between endometrioid carcinoma and control samples).

When the tissue sections were stained with UEAI lectin alone, blood vessels were found to be strongly positive both in normal and cancerous tissues, while epithelial cells were more often positive in cancer samples (Fig. 3f, i). The staining in cancer was also stronger and more often found in cytoplasm. All the UEAI-glycodelin positive cancer samples were also UEAI-staining positive.

Almost all $(86.5 \%)$ carcinoma samples were PR positive and the frequency of PR positive samples was similar irrespective of whether the samples were glycodelin or UEAI-glycodelin positive or negative ( $p>0.26$ for both). In the endometrioid carcinoma samples, frequencies of glycodelin, UEAI-glycodelin, and UEAI-staining positive glycodelin staining (with antibody) (d, g), UEAI-glycodelin staining (proximity-ligation based detection with glycodelin antibody and UEAI lectin) (e, h) and staining of UEAI lectin-reactive glycans (UEAI lectin staining) (f, i) in normal endometrium (d-f) and endometrial carcinoma $(\mathbf{g}-\mathbf{i})$ TMA samples. Scale in $\mathbf{a}$ is $100 \mu \mathrm{m}$ and the same scale applies to $\mathbf{c}-\mathbf{i}$. Scale in $\mathbf{b}$ is $200 \mu \mathrm{m}$.

samples were not statistically different between low (grades 1 and 2) and high (grade 3) grade endometrioid tumors (Table 2). Likewise, no differences in the frequency of positively-stained samples were found between small (tumor diameter $<2 \mathrm{~cm}$ ) and large tumors (diameter $\geq 2 \mathrm{~cm}$ ) or whether lymphovascular invasion was observed (for all $p>0.49$ ). Local carcinomas (stage I) were not different, in respect of staining frequencies, from those showing spread beyond the uterine corpus (stages II-IV).

\section{Discussion}

Glycodelin represents one of the foremost examples on how glycosylation dictates the function of a glycoprotein $[1,2,8,10,18]$. Although protein glycosylation is often altered in cancer [29-32] nothing is known about the glycosylation of glycodelin in endometrial carcinoma. Here, we describe a novel method for histochemical staining of a specific glycodelin-glycoform, based on in situ proximity ligation with a lectin and antibody. With this method we showed that glycodelin is differentially glycosylated in 
endometrial carcinoma, as compared with normal endometrium.

In order to establish a specific histochemical staining method for glycodelin expressed in endometrial carcinoma, we first carried out glycomics analysis of glycodelin expressed in the human endometrial carcinoma cell line HEC-1B. Our aim was to ascertain how the glycomic profiles differed from those reported for first trimester GdA and to use that information to support the selection of lectins for further studies. To this end, glycomics analysis showed that the major N-glycans of HEC-1B Gd exhibited decreased abundance of sialic acid (NeuAc) residues and carried only very low levels of Sda containing glycans (none in the highmass region), abundantly expressed in GdA, especially on larger glycans [7]. On the contrary, HEC-1B Gd N-glycans exhibited increased abundance of linear poly-LacNAc units (two or three LacNAcs) on their antennae, which were mainly undecorated, or to a lesser extent further modified by a NeuAc or fucose residue. This is further supported by the report showing that $\beta 1-4$-galactosyltransferase, which is involved in poly-LacNAc synthesis, is increased in endometrial cancer [42]. These differences are not likely to be explained by variations observed in GdA preparations isolated from endometrium and amniotic fluid of different individuals and during different times of pregnancy [43].

Next, we tested lectins that, based on the above glycomics analysis, were predicted to differentiate between carcinoma associated HEC-1B Gd and normal endometrium GdA. Decreased sialylation of HEC-1B Gd as compared with GdA suggested that SNA may differentiate between these two glycodelins and, indeed, SNA did not bind to HEC-1B Gd, while strong reaction with GdA was observed. Similarly, more abundant LacNAc epitopes in HEC-1B Gd agreed with increased ECL binding. However, it was somewhat surprising that LEL, DSL, and STL lectins, which should also bind poly-LacNAc [44], did not react well with HEC-1B Gd or GdA. Negative results are not necessarily informative as they may reflect low-binding affinity of the lectin in the assay conditions, rather than indicate genuine lack of the given carbohydrate epitope. Moreover, as the lectin binding was determined with a sandwich type of an assay, using glycodelin-specific monoclonal antibody and lectins, we could not rule out possible sterical hindrance preventing the binding of both the antibody and some of the lectins.

UEAI, a lectin that has been found to strongly recognize $\mathrm{H}$ type epitopes [45, 46], was found to bind well HEC-1B Gd. Indeed, MALDI-TOF/TOF MS/MS analysis showed a minor marker fragment ion corresponding to a fucose residue attached to a terminal galactose residue $(\mathrm{m} / \mathrm{z} 433$, Fig. 1c), suggesting the presence of $\mathrm{H}$ type antigen on elongated LacNAc antennae on the HEC-1B Gd. UEAI lectin also reacts with other fucosylated epitopes, such as
$\mathrm{Le}^{\mathrm{y}}$ and $\mathrm{Le}^{\mathrm{x}}$ epitopes [45-47]. However, UEAI did not show any significant binding to GdA. Since GdA exhibits Le $^{\mathrm{x}}$ antigens [9], binding to it should have been expected to some extent [5, 7, 9]. As fucosylated glycans in GdA are more prevalent in the Asn-63 glycosylation site [9], it is possible that the binding of UEAI to this glycosylation site could be blocked by the antibody binding in close proximity.

We have previously characterized several glycodelin isoforms, which differ especially in the level of sialylation and abundance of glycans with Sda epitopes [5, 7, 9], and have different effects on the viability of PBMCs. This may be due to reduced sialic acid content, as desialylation of GdA was found to abolish the immunomodulatory activity. Thus, it was somewhat surprising that HEC-1B Gd, which also appears to contain less sialic acids than GdA, had inhibitory activity on PBMC viability similar to GdA. However, as several different glycans in GdA are sialylated, it is possible that only some of those, which are also present in HEC-1B Gd, mediate the antiproliferative effect of glycodelin on PBMC or that different glycans can produce similar effect. Using enzymatic deglycosylation and/or differentially glycosylated glycodelin isoforms, we have found, that in addition to PBMC proliferation, the inhibitory activity of GdA on trophoblast invasion is dependent on glycosylation [16]. Again, despite the differences in glycosylation between GdA and HEC-1B Gd, both of these glycodelin glycoforms inhibited trophoblast invasion in similar potency.

After establishing, by glycan-analyses and lectin-binding studies, that HEC-1B endometrial carcinoma cells produce glycodelin with different $\mathrm{N}$-glycans than normal endometrium, we studied whether this can be seen also in endometrial carcinoma tissues. This is feasible as glycosylation of endometrial proteins is not only dependent on the phase of the menstrual cycle and menopausal status, but is further changed in malignant endometrium [48]. First, we studied glycodelin expression in normal endometrium and endometrial carcinoma. Previous studies have shown that high glycodelin expression is restricted to secretory endometrium, while expression in proliferative phase or postmenopausal endometrium is very low if detectable at all [1]. In order to detect also low levels of glycodelin, we used high antibody concentration, which stained normal secretory endometrium very strongly. Immunoreactive glycodelin was not often found in TMA samples, mostly obtained from postmenopausal women, and, apart from the few TMA samples representing normal secretory endometrium, the staining was weaker than that observed in control histological sections of normal secretory endometrium. Relatively strong glycodelin immunoreactivity was found in some samples, but there was no difference in frequencies of such samples between normal and endometrioid carcinoma 
groups. However, weak and focal glycodelin staining was observed more frequently in carcinoma samples. Interestingly, in few carcinoma samples containing adjacent histologically normal secretory epithelium, strong staining was observed only in the latter, suggesting that glycodelin expression may be reduced during malignant transformation. Previously most studies have not found glycodelin in cancerous endometrium [1]. One group has reported frequent glycodelin staining in endometrial cancer, which appears to be somewhat dependent on the used antibody [49]. Of note, glycodelin transcripts (mRNA) are more abundant in normal uterus than in uterine carcinomas [IST Online $^{\circledR}$ ver. 2.1.3 database, containing gene expression data of about 20,000 samples, http://ist.medisapiens.com/ [50]]. Glycodelin is also expressed in ovarian and breast cancers, where the levels are reduced in less differentiated tumors [25-27].

We found more frequent and stronger UEAI staining in endometrial cancer than in normal endometrium. Previous lectin studies have also shown that the binding of UEAI and its glycan epitope, $\mathrm{H}$ type antigen, are increased in endometrial carcinoma [51-53]. This corroborates with other reports showing that in endometrial carcinoma there is an increased expression of fucosyltransferases responsible for the expression of fucosylated epitopes including $\mathrm{H}$ type antigen [54, 55]. Also, lectin histochemistry on breast cancer tissues has shown a major reduction of UEAI staining after endo- $\beta$-galactosidase digestion, suggesting that UEAI lectin on breast cancer tissues recognizes fucosylated antigens on elongated LacNAc antennae of $\mathrm{N}$-glycans [56].

As the UEAI lectin binds HEC-1B Gd, but not GdA, and UEAI staining was stronger in endometrium carcinoma than in normal tissue, we next studied whether the specific UEAI reactive glycodelin (UEAI-glycodelin) would be more abundant in endometrial carcinoma than in normal endometrium. To this end, we established an in situ proximity ligation assay [57] with a combination of glycodelin antibody and UEAI lectin. A similar combination of an antibody and a lectin has previously been used for proximityligation assay for serum and tissue lysate samples [58] and an in situ approach has been used with a combination of protein- and glycan-specific antibodies [59, 60]. However, to our knowledge this is the first report for such a proteinglycoform-specific histochemical staining with a lectinantibody combination.

UEAI-glycodelin staining was almost exclusively found in endometrial carcinoma tissues and it was more frequent than the glycodelin staining. This is in keeping with the strong binding of UEAI to HEC-1B Gd, while no significant binding to GdA was observed. The staining was mostly found in luminal part, which suggests that the UEAI reactive glycans are mature only at the late stage of the secretory pathway. As proximity-ligation is a very sensitive technique, all glycodelin positive samples would have been expected to be positive also in UEAI-glycodelin staining. However, this was not the case. Thus, it is possible that in carcinoma samples glycodelin does not always carry UEAI reactive glycans. It has to be also noted that the tissue cores in TMA were quite small and the stained sections were not consecutive. Therefore, the pairwise comparison may not tell the whole truth. The lack of the glycodelin staining in some of the UEAI-glycodelin positive samples may be related to the same fact and a less sensitive method. Another possibility is the lack of specificity in the UEAI-glycodelin staining. However, this is unlikely as staining was absent both in blood vessels showing very strong UEAI reactivity (but no glycodelin staining) and in normal secretory endometrium samples containing high level of glycodelin.

The staining of UEAI-glycodelin appeared not to be related to tumor aggressiveness because significant associations with grade, stage, size, or lymphovascular invasion were not observed. An earlier study has shown that glycodelin levels are elevated in plasma and uterine flushings in postmenopausal women with endometrial adenocarcinoma, as compared with those without neoplasia [61]. Since UEAIreactive glycodelin was found, with one exception, only in carcinoma samples, it is likely that its circulating levels would be more specific for detection of endometrial cancer than those of total glycodelin. However, whether UEAI-glycodelin, as an apparent neoantigen in endometrial carcinoma, is a suitable marker for cancer detection, or target for vaccination strategies [62] or cancer treatment, remains to be studied.

In conclusion, using mass spectrometry glycomics strategies and lectins, we found that glycodelin expressed in HEC-1B human endometrial carcinoma cell line is differentially glycosylated as compared with normal decidualized endometrium-derived glycodelin. Despite of the differences in glycosylation these glycodelin isoforms had similar inhibitory activity on trophoblast cell invasion and PBMC proliferation, activities that we have previously found to be associated with specific glycosylation of glycodelin. We, furthermore, established a novel in situ proximity ligation assay with a lectin and an antibody, and showed that glycodelin is differentially glycosylated in endometrial carcinoma tissues as compared with normal endometrium. Whether specific detection of such glycodelin in endometrial carcinoma has any clinical significance remains to be established. Similar approach and methods can be used for studies of other glycoproteins.

Acknowledgements The authors thank Ms Annikki Löfhjelm for excellent technical assistance. This work was supported by the grants from the Academy of Finland (HK), Sigrid Jusélius Foundation (HK), Finnish Cancer Foundation (HK), Helsinki University Hospital research funds (ML) and Biotechnology and Biological Sciences Research Council (grant number BB/K016164/1 (AD and SMH). 


\section{Compliance with ethical standards}

Conflict of interest The authors declare that they have no conflict of interest.

Publisher's note Springer Nature remains neutral with regard to jurisdictional claims in published maps and institutional affiliations.

Open Access This article is licensed under a Creative Commons Attribution 4.0 International License, which permits use, sharing, adaptation, distribution and reproduction in any medium or format, as long as you give appropriate credit to the original author(s) and the source, provide a link to the Creative Commons license, and indicate if changes were made. The images or other third party material in this article are included in the article's Creative Commons license, unless indicated otherwise in a credit line to the material. If material is not included in the article's Creative Commons license and your intended use is not permitted by statutory regulation or exceeds the permitted use, you will need to obtain permission directly from the copyright holder. To view a copy of this license, visit http://creativecommons. org/licenses/by/4.0/.

\section{References}

1. Seppälä M, Taylor RN, Koistinen H, Koistinen R, Milgrom E. Glycodelin: a major lipocalin protein of the reproductive axis with diverse actions in cell recognition and differentiation. Endocr Rev. 2002;23:401-30.

2. Seppälä M, Koistinen H, Koistinen R, Hautala L, Chiu PC, Yeung WS. Glycodelin in reproductive endocrinology and hormonerelated cancer. Eur J Endocrinol. 2009;160:121-33.

3. Seppala M, Koistinen H, Koistinen R, Chiu PC, Yeung WS. Glycosylation related actions of glycodelin: gamete, cumulus cell, immune cell and clinical associations. Hum Reprod Update. 2007;13:275-87.

4. Yeung WS, Lee KF, Koistinen R, Koistinen H, Seppala M, Ho PC, et al. Glycodelin: a molecule with multi-functions on spermatozoa. Soc Reprod Fertil Suppl. 2007;63:143-51.

5. Lee CL, Pang PC, Yeung WS, Tissot B, Panico M, Lao TT, et al. Effects of differential glycosylation of glycodelins on lymphocyte survival. J Biol Chem. 2009;284:15084-96.

6. Yeung WS, Lee KF, Koistinen R, Koistinen H, Seppala M, Chiu PC. Effects of glycodelins on functional competence of spermatozoa. J Reprod Immunol. 2009;83:26-30.

7. Lee CL, Chiu PC, Pang PC, Chu IK, Lee KF, Koistinen R, et al. Glycosylation failure extends to glycoproteins in gestational diabetes mellitus: evidence from reduced alpha2-6 sialylation and impaired immunomodulatory activities of pregnancy-related glycodelin-A. Diabetes. 2011;60:909-17.

8. Pang PC, Haslam SM, Dell A, Clark GF. The human fetoembryonic defense system hypothesis: twenty years on. Mol Aspects Med. 2016;51:71-88.

9. Dell A, Morris HR, Easton RL, Panico M, Patankar M, Oehniger $\mathrm{S}$, et al. Structural analysis of the oligosaccharides derived from glycodelin, a human glycoprotein with potent immunosuppressive and contraceptive activities. J Biol Chem. 1995;270:24116-26.

10. Morris HR, Dell A, Easton RL, Panico M, Koistinen H, Koistinen $\mathrm{R}$, et al. Gender-specific glycosylation of human glycodelin affects its contraceptive activity. J Biol Chem. 1996;271: 32159-67.

11. Chiu PC, Chung MK, Tsang HY, Koistinen R, Koistinen H, Seppala M, et al. Glycodelin-S in human seminal plasma reduces cholesterol efflux and inhibits capacitation of spermatozoa. J Biol Chem. 2005;280:25580-9.
12. Chiu PC, Koistinen R, Koistinen H, Seppala M, Lee KF, Yeung WS. Binding of zona binding inhibitory factor-1 (ZIF-1) from human follicular fluid on spermatozoa. J Biol Chem. 2003;278:13570-7.

13. Chiu PC, Chung MK, Koistinen R, Koistinen H, Seppala M, Ho $\mathrm{PC}$, et al. Cumulus oophorus-associated glycodelin-C displaces sperm-bound glycodelin-A and -F and stimulates spermatozoazona pellucida binding. J Biol Chem. 2007;282:5378-88.

14. Lee CL, Lam KK, Vijayan M, Koistinen H, Seppala M, Ng EH, et al. The pleiotropic effect of glycodelin-A in early pregnancy. Am J Reprod Immunol. 2016;75:290-7.

15. Morrow DM, Xiong N, Getty RR, Ratajczak MZ, Morgan D, Seppala M, et al. Hematopoietic placental protein 14. An immunosuppressive factor in cells of the megakaryocytic lineage. Am J Pathol. 1994;145:1485-95.

16. Lam KK, Chiu PC, Chung MK, Lee CL, Lee KF, Koistinen R, et al. Glycodelin-A as a modulator of trophoblast invasion. Hum Reprod. 2009;24:2093-103.

17. Lunghi L, Ferretti ME, Medici S, Biondi C, Vesce F. Control of human trophoblast function. Reprod Biol Endocrinol. 2007;5:6.

18. Lapid K, Sharon N. Meet the multifunctional and sexy glycoforms of glycodelin. Glycobiology. 2006;16:39R-45R.

19. Koistinen H, Seppala M, Nagy B, Tapper J, Knuutila S, Koistinen R. Glycodelin reduces carcinoma-associated gene expression in endometrial adenocarcinoma cells. Am J Obstet Gynecol. 2005;193:1955-60.

20. Hautala LC, Koistinen R, Seppala M, Butzow R, Stenman UH, Laakkonen P, et al. Glycodelin reduces breast cancer xenograft growth in vivo. Int J Cancer. 2008;123:2279-84.

21. Hautala LC, Koistinen R, Koistinen H. Repressed PKCdelta activation in glycodelin-expressing cells mediates resistance to phorbol ester and TGFbeta. Cell Signal. 2016;28:1463-9.

22. Uchida H, Maruyama T, Nagashima T, Asada H, Yoshimura Y. Histone deacetylase inhibitors induce differentiation of human endometrial adenocarcinoma cells through up-regulation of glycodelin. Endocrinology. 2005;146:5365-73.

23. Ohta K, Maruyama T, Uchida H, Ono M, Nagashima T, Arase T, et al. Glycodelin blocks progression to $\mathrm{S}$ phase and inhibits cell growth: a possible progesterone-induced regulator for endometrial epithelial cell growth. Mol Hum Reprod. 2008;14:17-22.

24. Li ZR, Hromchak R, Mudipalli A, Bloch A. Tumor suppressor proteins as regulators of cell differentiation. Cancer Res. 1998;58:4282-7.

25. Mandelin E, Lassus H, Seppala M, Leminen A, Gustafsson JA, Cheng $\mathrm{G}$, et al. Glycodelin in ovarian serous carcinoma: association with differentiation and survival. Cancer Res. 2003;63:6258-64.

26. Shabani N, Mylonas I, Kunert-Keil C, Briese V, Janni W, Gerber $\mathrm{B}$, et al. Expression of glycodelin in human breast cancer: immunohistochemical analysis in mammary carcinoma in situ, invasive carcinomas and their lymph node metastases. Anticancer Res. 2005;25:1761-4.

27. Hautala LC, Greco D, Koistinen R, Heikkinen T, Heikkila P, Aittomaki K, et al. Glycodelin expression associates with differential tumour phenotype and outcome in sporadic and familial non-BRCA1/2 breast cancer patients. Breast Cancer Res Treat. 2011;128:85-95.

28. Varki A. Biological roles of oligosaccharides: all of the theories are correct. Glycobiology. 1993;3:97-130.

29. Hakomori S. Aberrant glycosylation in tumors and tumorassociated carbohydrate antigens. Adv Cancer Res. 1989;52: 257-331.

30. Varki A, Kannagi R, Toole B, Stanley P. Glycosylation changes in cancer. In: Varki A, Cummings RD, Esko JD, Stanley P, Hart GW, Aebi M, et al., editors. Essentials of glycobiology. 3rd ed. Cold Spring Harbor, NY: Cold Spring Harbor Laboratory Press; 2017. Chapter 47. https://www.ncbi.nlm.nih.gov/books/NBK453023/. 
31. Pinho SS, Reis CA. Glycosylation in cancer: mechanisms and clinical implications. Nat Rev. 2015;15:540-55.

32. Stowell SR, Ju T, Cummings RD. Protein glycosylation in cancer. Annu Rev Pathol. 2015;10:473-510.

33. Kuramoto H, Hamano M, Imai M. HEC-1 cells. Hum Cell. 2002;15:81-95.

34. Kuramoto H, Tamura S, Notake Y. Establishment of a cell line of human endometrial adenocarcinoma in vitro. Am $\mathrm{J}$ Obstet Gynecol. 1972;114:1012-9.

35. Riittinen L, Narvanen O, Virtanen I, Seppala M. Monoclonal antibodies against endometrial protein PP14 and their use for purification and radioimmunoassay of PP14. J Immunol Methods. 1991;136:85-90.

36. Koistinen H, Koistinen R, Dell A, Morris HR, Easton RL, Patankar MS, et al. Glycodelin from seminal plasma is a differentially glycosylated form of contraceptive glycodelin-A. Mol Hum Reprod. 1996;2:759-65.

37. North SJ, Jang-Lee J, Harrison R, Canis K, Ismail MN, Trollope A, et al. Mass spectrometric analysis of mutant mice. Methods Enzymol. 2010;478:27-77.

38. Ceroni A, Maass K, Geyer H, Geyer R, Dell A, Haslam SM. GlycoWorkbench: a tool for the computer-assisted annotation of mass spectra of glycans. J Proteome Res. 2008;7:1650-9.

39. Trundley A, Gardner L, Northfield J, Chang C, Moffett A. Methods for isolation of cells from the human fetal-maternal interface. Methods Mol Med. 2006;122:109-22.

40. Lee C-L, Vijayan M, Wang X, Lam KKW, Koistinen H, Seppala $\mathrm{M}$, et al. Glycodelin-A stimulates the conversion of human peripheral blood CD16-CD56bright NK cell to a decidual NK celllike phenotype. Hum Reprod. 2019;34:689-701.

41. Pasanen A, Tuomi T, Isola J, Staff S, Bützow R, Loukovaara M. L1 cell adhesion molecule as a predictor of disease-specific survival and patterns of relapse in endometrial cancer. Int J Gynecol Cancer. 2016;26:1465-71.

42. Kubushiro K, Ma J, Fukuchi T, Banno K, Muramatsu Y, Tsukazaki K, et al. Changes of beta-1,4-galactosyltransferase with the development of endometrial cancer. Gynecol Obstet Invest. 1999;48:211-4.

43. Koistinen H, Easton RL, Chiu PC, Chalabi S, Halttunen M, Dell A, et al. Differences in glycosylation and sperm-egg binding inhibition of pregnancy-related glycodelin. Biol Reprod. 2003;69:1545-51.

44. Cummings RD, Darvill AG, Etzler ME, Hahn MG. Glycanrecognizing probes as tools. In: Varki A, Cummings RD, Esko JD, Stanley P, Hart GW, Aebi M, et al., editors. Essentials of glycobiology. 3rd ed. Cold Spring Harbor, NY: Cold Spring Harbor Laboratory Press; 2017. Chapter 48. https://www.ncbi.nlm.nih. gov/books/NBK453096/.

45. Baldus SE, Thiele J, Park YO, Hanisch FG, Bara J, Fischer R. Characterization of the binding specificity of Anguilla anguilla agglutinin (AAA) in comparison to Ulex europaeus agglutinin I (UEA-I). Glycoconj J. 1996;13:585-90.

46. Mollicone R, Cailleau A, Imberty A, Gane P, Perez S, Oriol R. Recognition of the blood group $\mathrm{H}$ type 2 trisaccharide epitope by 28 monoclonal antibodies and three lectins. Glycoconj J. 1996;13:263-71.

47. Molin K, Fredman P, Svennerholm L. Binding specificities of the lectins PNA, WGA and UEA I to polyvinylchloride-adsorbed glycosphingolipids. FEBS Lett. 1986;205:51-5.
48. Clark GF. Functional glycosylation in the human and mammalian uterus. Fertil Res Pract. 2015;1:17.

49. Lenhard M, Heublein S, Kunert-Keil C, Vrekoussis T, Lomba I, Ditsch N, et al. Immunosuppressive Glycodelin A is an independent marker for poor prognosis in endometrial cancer. BMC Cancer. 2013;13:616.

50. Kilpinen S, Autio R, Ojala K, Iljin K, Bucher E, Sara H, et al. Systematic bioinformatic analysis of expression levels of 17,330 human genes across 9,783 samples from 175 types of healthy and pathological tissues. Genome Biol. 2008;9:R139.

51. Inoue M, Sasagawa T, Saito J, Shimizu H, Ueda G, Tanizawa O, et al. Expression of blood group antigens A, B, H, Lewis-a, and Lewis-b in fetal, normal, and malignant tissues of the uterine endometrium. Cancer. 1987;60:2985-93.

52. Aoki D, Nozawa S, Iizuka R, Kawakami H, Hirano H. Differences in lectin binding patterns of normal endometrium and endometrial adenocarcinoma, with special reference to staining with Ulex europeus agglutinin 1 and peanut agglutinin. Gynecol Oncol. 1990;37:338-45.

53. Toda T, Sadi AM, Egawa H, Atari E, Qureshi B, Nagai Y. Affinity of four lectins for endocervical and endometrial nonneoplastic and neoplastic glandular epithelium. Histopathology. 1998;32:257-63.

54. Wang JW, Ambros RA, Weber PB, Rosano TG. Fucosyltransferase and alpha-L-fucosidase activities and fucose levels in normal and malignant endometrial tissue. Cancer Res. 1995;55:3654-8.

55. Skovlund VR. ABH and related histo-blood group antigens in normal \& malignant human endometrium in relation to genetic and hormonal factors. APMIS Suppl. 1997;69:1-33.

56. Ito $\mathrm{N}$, Imai $\mathrm{S}$, Haga $\mathrm{S}$, Nagaike $\mathrm{C}$, Morimura $\mathrm{Y}$, Hatake $\mathrm{K}$. Localization of binding sites of Ulex europaeus I, Helix pomatia and Griffonia simplicifolia I-B4 lectins and analysis of their backbone structures by several glycosidases and poly-Nacetyllactosamine-specific lectins in human breast carcinomas. Histochem Cell Biol. 1996;106:331-9.

57. Söderberg O, Gullberg M, Jarvius M, Ridderstråle K, Leuchowius K-J, Jarvius J, et al. Direct observation of individual endogenous protein complexes in situ by proximity ligation. Nat Methods. 2006;3:995-1000.

58. Oliveira FMS, de, Mereiter S, Lönn P, Siart B, Shen Q, Heldin J, et al. Detection of post-translational modifications using solid-phase proximity ligation assay. New Biotechnol. 2018;45:51-9.

59. Conze T, Carvalho AS, Landegren U, Almeida R, Reis CA, David $\mathrm{L}$, et al. MUC2 mucin is a major carrier of the cancer-associated sialyl-Tn antigen in intestinal metaplasia and gastric carcinomas. Glycobiology. 2010;20:199-206.

60. Pinto R, Carvalho AS, Conze T, Magalhães A, Picco G, Burchell $\mathrm{JM}$, et al. Identification of new cancer biomarkers based on aberrant mucin glycoforms by in situ proximity ligation. J Cell Mol Med. 2012;16:1474-84.

61. Li TC, Okon MA, Dalton CF, Heatley M, Laird SM. Is the measurement of placental protein-14 and CA-125 in plasma and uterine flushings useful in the evaluation of perimenopausal and post-menopausal bleeding? Hum Reprod. 1998;13:2895-901.

62. Krasnova L, Wong CH. Exploring human glycosylation for better therapies. Mol Aspects Med. 2016;51:125-43. 